MODELING, IDENTIFICATION AND CONTROL, 1994, vOL. 15, NO. 3, 127-134

doi:10.4173/mic.1994.3.1

\title{
The future of control
}

\author{
K. J. ÅSTRÖM \\ Keywords: Automatic control, history, theory, applications
}

This paper gives a personal view of the emergence, development and future of automatic control. The assessment is that automatic control has been very successful, but that we are now at a crossroads where we have to decide if we want to take an holistic view with full systems responsibility or if we want the field to fracture into a collection of subspecialities.

\section{Introduction}

This paper presents some personal reflections on the field of automatic control. A broad view of the field is given first, followed by a very brief description of its development. The overview of the main areas presented in $\S 4$ gives a basis for the discussion of the future in $\S 5$.

\section{Our field}

Research and education in engineering were originally focused on specific technologies such as mining, machines, electricity and radio techniques. This led to organization of engineering schools with departments such as mining, mechanical engineering, electrical engineering, aeronautics, etc. This served very well in the beginning of this century. The situation changed significantly with the advent of fields like automatic control, that are pervasive and cross traditional department boundaries.

Automatic control is crucial for generation and transmission of energy, process control, manufacturing, communication, transportation and entertainment. It has been a key element in the design of experimental equipment and instrumentation used in basic sciences. Principles of automatic control are also having an impact on such diverse fields as biology and economics.

Automatic control, like many other branches of engineering science, has developed in the same pattern as natural science. Although there are strong similarities between natural science and engineering science it is important to realize that there are some fundamental differences. The inspiration for natural science is to understand phenomena in nature. This has led to a strong emphasis on analysis and isolation of simple phenomena, an extreme case being particle physics. A key goal is to find basic laws that describe nature. The inspiration of engineering science is to understand, invent and design man-made technical systems. This places much more emphasis on design. It is natural to consider interaction instead of isolation, and a key goal is to find principles that are useful when dealing with complex systems. Feedback which is at the heart of automatic control is a good example of a systems principle.

The wide applicability of automatic control has many advantages, but it has also created some difficulties. Since automatic control can be used in so many different

Received 6 April 1994.

†Department of Automatic Control, Lund Institute of Technology, Lund, Sweden. 
fields, it is a very good vehicle for technology transfer. A key difficulty is, however, the positioning of the field academically. One possibility is to have separate control groups within traditional departments of electrical, mechanical and chemical engineering, another is to have a central group with global responsibility. One advantage with the centralized approach is the ease of transfer of ideas among different fields. A disadvantage is that it is more difficult to maintain the expertise of different application areas which is so essential. Specific process knowledge is indeed indispensable to successful use of automatic control. Personally I am in favour of the centralized approach that is used in both Trondheim and Lund.

In summary, automatic feedback control is a young exciting field. It has found widespread application in the modern technological society, many systems cannot be built without feedback. There is a dynamic development with many challenging problems remaining to be solved.

\section{Past and present}

Although control devices have been used for a very long time it is reasonable to say that the field of automatic control emerged as a discipline during the second World War when it was realized that science could have a drastic impact on the war effort. The development was strongly stimulated by the cross disciplinary teams that were formed. The Servomechanisms Laboratory at MIT, which was established in 1940 under the leadership of Professor Gordon S. Brown, can be viewed as the US cradle of automatic control, although much work on control was also done at the neighbouring Radiation Laboratory and Lincoln Laboratory, see Gallager and Mitter (1990).

\subsection{How the field emerged?}

Automatic control emerged as a fusion of ideas from process control, flight control and feedback amplifiers. A common theory and a methodology to solve engineering problems were developed. By abstraction, a wide range of problems could be dealt with in a common framework called servomechanism theory. At that time automatic control included a graphical representation that is independent of the technological details, a mathematical theory based on linearization, complex variables and Laplace transforms, a methodology for determining transfer functions experimentally and techniques for designing feedback laws. The methodology was supported by a unified approach for implementing controllers using analogue techniques, supplemented by analogue computers for simulation of the systems.

\subsection{The second wave}

Automatic control was very well established in the beginning of the 1960's in the form of servomechanism theory, with a wide range of applications. The field received a substantial injection of ideas from several sources. Computers started to be used for implementation of control systems, the space effort posed new challenging problems and there was an influx of ideas from mathematics. This led to new developments in the field with contributions such as state space theory, optimal control, stochastic control, sampled data systems and nonlinear control theory. Initially there was a lot of controversy between 'classical' and 'modern' control but opinion is now largely united and most professionals agree that it is valuable to have several views of a control problem. 


\subsection{Education}

Much of the early work was done in laboratories associated with the war effort in England, USA, and USSR. Automatic control was a very powerful technique that could be applied to many problems in diverse fields. It quickly received world wide acceptance in industry and academia. Control groups were created in many companies, new industrial enterprises specializing in control were established. Courses in the field were introduced at practically all engineering schools. In Sweden a teaching position (speciallärare) was established at KTH in 1949 followed by a professorship in 1959. Education at Chalmers started in 1959 with a donation from Källe, one of the new Swedish control companies. A regular professorship was established in 1962. The chair of automatic control in Lund was established in 1964, it was later followed by positions in Uppsala, Linköping and Luleå.

\subsection{Organization}

The early work in automatic control was to a large extent classified. Many researchers felt that there were strong benefits from an open exchange of ideas which resulted in a strong drive for internationalization. In 1956 there were plans for no less than eight national meetings on automatic control in Europe. Good leadership resulted in the formation of an international body IFAC (International Federation of Automatic Control) which became the international forum for our field, see Chestnut (1982) and Luoto (1978). An organizational structure was set up, triannual World Congresses, symposia and workshops were organized and a journal were started. The first World Congress held in Moscow in 1960 was probably the first manifestation of Automatic Control as an established field. The activities have expanded substantially and today there is an IFAC meeting almost every week. There are also other bodies that are important in our field. The Automatic Control Council in the USA organizes the yearly American Control Conference in collaboration with many engineering societies. A European Control Conference which meets every other year started with a meeting in Grenoble in 1991. An Asian Control Conference will have its first meeting in 1994. Automatic control has an important place in the meetings of many engineering societies. Establishing journals are an important aspect of a scientific field. A number of high quality journals appeared simultaneously with the emergence of organizations.

\section{What is automatic control?}

One way to find the factual content of a field and how it has developed is to investigate the contents of the leading conferences in the field over a number of years. Analysing the contents of the IFAC World Congresses, the American Control Conference and the IEEE Decision and Control Conference over the period 1960 to 1993 the following picture emerges.

\subsection{System theory}

In practically all meetings in the period there are sessions on linear systems (time invariant as well as time varying), nonlinear systems, stochastic systems, discrete time systems, time delay systems, distributed parameter systems, and decentralized systems. Occasionally there has also been interest in special topics such as variable structure systems. These sessions naturally have a strong flavour of applied mathematics. The issues discussed are representations, development of concepts such as stability, 
observability, controllability, analysis of structure etc. The tools used are typically ordinary and partial differential equations but also algebra and geometry. Lately there has been a significant interest also in differential algebraic systems, sometimes under the label of 'behavioural systems' and in discrete event dynamical systems. The field of linear systems has been declared uninteresting at many instances but interest has often been renewed due to new viewpoints. Lately there has been a significant interest in applying ideas from differential geometry to nonlinear systems.

\subsection{Modeling and identification}

Models are a very important element of automatic control. Modeling is a significant portion of the development of all control systems. It is also an area where specific process knowledge play a major role. There are two approaches to modeling, one is based on physics (white box modeling) the other on plant experiments (black box modeling). It is also possible to combine the approaches. This has recently been popularized under the name of grey box identification. The early effort was focused on experimental techniques based on frequency response. Lately there has been a significant effort in the fertile field of system identification. The area of model reduction is a closely related field which keeps popping up in meetings.

\subsection{Design theories}

It is difficult to capture design problems formally because there are so many factors to consider. A common approach has been to formalize one or a few aspects of the problem so that it can be dealt with mathematically. A lot of creativity has been demonstrated in this area. The early work has a strong engineering flavour with emphasis on graphical methods like loop shaping and root locus. Analytical design methods such as pole placement were introduced later. Optimization is a natural tool which has stimulated much research on optimal control. It comes in many flavours, LQG, LTR, dynamic programming and the maximum principle. Because of the nature of the problem and the calibre of the people involved much interesting mathematics has also appeared. A focus on disturbances led to the development of stochastic control theory. In the last decade there has been a significant effort to consider model uncertainty. This resulted in robust control techniques like QFT, $H_{\infty}, L_{1}$-methods and $\mu$. Again there has lately been a significant interest in nonlinear methods such as feedback linearization.

Finding fundamental limitations to achievable performance is a fundamental aspect of control system design. The early work by Bode gave considerable insight into this problem, but it is an important area that deserves much more research.

Control system design is a difficult area. If all engineering aspects are considered the problem becomes very messy. If the problem is simplified so that it can be solved elegantly many aspects have to be neglected. This has caused much controversy. It will probably be a long time before we have good design tools that will deal with the real issues of control system design. An important requirement is that the methods should also be devcloped and packaged so that they can be used by an engineer with training at the masters level.

An indication of the importance of having techniques that can be used by persons with a moderate technical background is that special design methods have appeared in applications area with very little initial contact with the main stream control theory. Typical examples are, dynamic matrix control (DMC), in chemical process control, and 
the computed torque method in robotics. A dynamic matrix is nothing but a truncated impulse response for a multivariable system. Since the chemical engineers were ignorant of this they solved the problem, invented a new vocabulary (DMC, QDMC, IDCOM). There is a good lesson to be learned from this. We must package our results so that they are accessible.

\subsection{Learning and adaptation}

To have systems that could automatically learn about a process and do control design automatically has been a long standing dream among control engineers. There have been sessions on this from the 1960 Moscow Conference onwards. This has led to the model reference adaptive controller and the self-tuning regulator. It is, however, only recently in the 1980 s that the simpler problem of providing control systems with devices for automatic tuning of the controller has been approached. This has had a significant impact, particularly in the field of process control. However, much work remains before the longstanding goal is fulfilled. There are strong connections to the problem of machine learning.

\subsection{Numerics and simulation}

Automatic control has been strongly linked to computing throughout its history. Computing is important both for analysis, simulation and implementation. Analogue computing was used initially but has lately been replaced by digital computing. The development of the computer is probably the factor that has changed the most in the short span of the field. An illustration of this is that at the Moscow Conference computing was presented under the subheading 'Pneumatic Components and Computing Devices' in the components category! The emergence of computers has also put much more emphasis on numerics, which is important both for analysis and implementation. Lately there have been both special sessions and special symposia on Computer Aided Control Engineering. The emergence of interactive software like

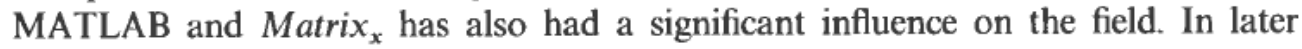
conferences we can find several special sessions on this. Simulation is another very important area which now has its own conferences and publications.

\subsection{Implementation}

Implementation is an important issue. Because of the rapid development of microelectronics it is also one of the factors that has changed the most over the past 50 years. At the Moscow Conference there were three major groups, theory, components and applications. Implementation issues came under components. Some aspects in implementation such as sampled data systems, are very well represented but many equally important aspects are not dealt with at all. Many important aspects on implementation are not covered in textbooks. Typical examples are integrator windup, real time kernels and man-machine interfaces. In my mind this is probably one of the factors that has contributed most to the infamous GAP between theory and practice.

\subsection{Education}

Education has been well represented at the conferences. The sessions have naturally been dominated by academics. Lately there have been efforts to include industrialists. 


\subsection{Applications}

The major application areas have not changed much over the years. At the Moscow Conference there were sessions on metalworking, electrical power systems, electrical drives, transport, industrial processes, chemical and oil, thermal and nuclear power and metallurgical processes. In later years we find applications in aerospace, automotive, microelectronics and home electronics (CD, video etc.). A long experience with journals and conferences has, however, shown that it is very difficult to get good applications papers. The engineers who really know about the applications do not have the time or the permission to publish. Many of those who do write have only a superficial knowledge about the applications. This sends distorted signals in all directions. There are occasional efforts with special issues of journals where really good applications papers sometimes appear. We need these to educate the next generation of engineers.

Perhaps the most interesting aspect of applications is when application of automatic control can lead to totally new processes. This is often manifested in new products, drastic decreases in cost or performance. There are many examples of this in the areas of flight control, mechatronics and power systems.

\subsection{Fads and fallacies}

Science is not free of fashions. A subfield may receive sudden interest because of some interesting new result or spectacular application. This will naturally attract attention from researchers and students which is later manifested by many papers, emergence of special sessions at conferences, special issues of journals, books and even attention in the media, particularly if the journalists can smell conspiracy, recent examples of this are neural networks, artificial intelligence and lately fuzzy control. In such cases it is easy to create controversy, particularly if research funding is at stake. Fuzzy control is a recent example where there is much publicity and many poor publications. It is frustrating that the papers are written in such a way that the results cannot be checked. There are, however, also cases where fuzzy control may be useful. There is clearly a need to sort out the field. This will not happen unless serious work is involved. There are also 'snake oil salesmen' $\uparrow$ around who claim that control problems can be solved without understanding anything about them.

\section{Our future}

Automatic control has emerged as an interesting and successful discipline in a period of about 50 years. The development of the field has been very dynamic. It is one of the first examples of a systems discipline that transcends the boundaries of the traditional engineering disciplines. Automatic control has a firm base in applied mathematics and an unusually wide range of applications. Automatic control is 'mission critical' for many things in modern life, for example in airplanes and CDplayers. We find control systems all around us. For many of us like Jens Balchen and myself it has been very rewarding to watch and participate in the development. A natural question to ask is if the development will continue or if it has peaked. Having a positive attitude I strongly believe in a continuation, but what happens will of course strongly depend on ourselves. I will discuss some factors below that may influence the development.

† Snakeoil - any of various concoctions of questionable medical value sold as an all-purpose curative especially by travelling hucksters. 


\subsection{Intellectual drivers}

The health of any discipline depends strongly on whether it can attract the brightest students. This is easy to find out at each university. Stimulating and challenging problems attract bright students. We must ensure that we are indeed working on those problems, there are lots of them in the field.

\subsection{Labels}

The importance of labels is obvious to anyone who sees how carefully the major companies watch them. In automatic control we use too many names for our discipline, control engineering, feedback control, systems science and cybernetics (kybernetikk in Norwegian). I think it is important to pick one word for our discipline and stick to it.

\subsection{Full system responsibility}

The boundaries of our field is a key question. I feel very strongly that automatic control is a systems discipline which means that we should take full systems responsibility. This means that we should educate students to be capable of solving the whole problem from conceptual design to implementation and commissioning. Control will fade away if we consider the job done when a mathematical description of the control law is obtained. This means that we need to teach about implementation!

\subsection{Balance of theory and applications}

The balance between theory and practice is a key issue. Throughout the short history of our field there has been a continuous and sometimes heated debate about the GAP between theory and practice. I personally believe that it is important to strike a balance. It is important to look inwards to improve our basic understanding, our theory and our design methods. But this is not enough, we must look at the applications. Lately there have been several instances where control engineers have been very slow in getting into an application area although we had been in a position to make major contributions. The following quote from John van Neumann gives a sobering thought: "As a mathematical discipline traveis far from its empirical source, or still more, if it is a second or third generation only indirectly inspired by ideas coming from reality, it is beset with very grave dangers. It becomes more and more aestheticising, more and more purely l'art pour l'art.... At a great distance from its source or after much 'abstract' inbreeding, a mathematical subject is in great danger of degeneration." If this applies to mathematics how about our own field?

\subsection{Compactification of knowledge}

Our discipline is very young scientifically. Courses have appeared in a partly random fashion and there is currently a lot of diversity. We need to take a careful look at our knowledge base and explore how it can be weeded out and streamlined to give a better education both for our own researchers but more importantly for students who only take one or two courses.

\subsection{Research funding}

It is natural that research agencies in a dynamic field have come to expect new catchy titles regularly. We must, however, realize that our field is maturing and that it may indeed be a very worthwhile effort to clean up fundamental problems even if they 
should come under the heading of linear or nonlinear systems. It is permissible in mathematics and physics, why not in control? In the same spirit it is probably also useful to exercise some care when promoting areas with a lot of hype.

\section{Conclusions}

Automatic control emerged as a discipline about 50 years ago and it has had a very dynamic development. It is one of the first systems disciplines to transcend the boundaries of traditional engineering fields. It has a wide range of applications which show up in all aspects of modern life and sometimes make it even more enjoyable. There are a large number of challenging problems ahead with the potential to attract the very best students. It is important that we accept the challenges and in particular that we are willing to deal with the full aspects including theory and applications.

\section{References}

Chestnut, H., Ed. (1982). Impact of Automatic Control-Present and Future. (VDI/VDEGesellschaft, Düsseldorf).

Luoto, U., Ed. (1978). 20 Years Old; 20 Years Young. An Anniversary Publication 1957-1977. Automatica, 14, 49-75.

GallaGer, R. G. and MitTeR, S. K. (1990). From Servo Loops to Fibre Nets. (The Laboratory for Information and Decision Systems. Massachusetts Institute of Technology, Cambridge, M.A). 